\title{
Constraint 3D Elastic Model for the Segmentation in Cardiac MRI
}

\author{
J Pousin ${ }^{1}$, Y Rouchdy ${ }^{1}$, M Picq ${ }^{1}$, J Schaerer ${ }^{2}$, P Clarysse $^{2}$ \\ ${ }^{1}$ Institute C Jordan INSA de Lyon, 69621 Villeurbanne cedex, France \\ ${ }^{2}$ CREATIS INSA de Lyon, 69621 Villeurbanne cedex, France
}

\begin{abstract}
In this paper a $3 D$ elastic model for the segmentation of cardiac magnetic resonance imaging has been proposed and analyzed. Elastic models for segmentation usually involve minimization of internal and external energy. A problem we observed with standard internal and external energy is that the local or the global reached minima do not impose the external energy to be zero. To eliminate this difficulty, we propose introducing a constraint. The constraint problem is proved to be mathematically well posed, and a simple algorithm which avoids computing the Lagrange multiplier is provided. This algorithm is proved to be convergent. Finally the efficiency of the method is shown with numerical experiments.
\end{abstract}

\section{Introduction}

Volumetric biomechanical models for segmenting cardiac medical images deals with a clinical Magnetic Resonance data set (M.R. volume data set). By image segmentation we refer to processes which identify all voxels which belong to a group sharing an homogeneity criterion (most often this criterion is the grey level). Segmentation is required for the identification of the object (i.e. the heart) in the M. R. volume data. Here, we deal with edge-based algorithms which try to detect the borderline of a structure, that is to say the discontinuity surfaces of the "gradient" of the grey level function $H$.

Generally segmentation proceeds in three stages [1]. Firstly, a mesh of a given anatomical structure is created, in our concern: a volumetric tetrahedra mesh of the heart including both left and right ventricules extracted from a databank. The second step, is at a coarse scale to applied successively a rigid transformation, and a similarity then later upgraded to a full affine transformation. The last stage concerns the fine scale. The minimization of internal and external energy permits more local deformations. This stage is important when biomechanical models aim to specify physiological important parameters. The goal of this article is to analyze how a biomechanical model evolves under the influence of both an internal energy computed from linear elasticity models representing physical properties of the organ and an external energy computed from the image as defined in the framework of deformable templates. A problem we observed with standard internal and external energy is that the reached local or global minima do not impose to the external energy to be zero (see Figure 1). That is a serious limitation when the external energy is derived with the Gradient Vector Flow method [5], since the zero level of the external energy is reached when the boundaries of the anatomical structure fit with the image edges. The remedies we propose is to introduce a constraint in order that the external energy is zero for a minimizer of the energy.

The outline of the paper is as follows. The minimization problem induced by a linear elastic model under constraint is introduced, and an existence result for a minimizer is proved. Then an algorithm for computing a minimizer is proposed. In section 3 a toy problem is considered for analyzing the convergence properties of the algorithm introduced previously. Finally, the paper is ended with numerical examples and heart patients images segmented with our method.

\section{Methods}

We recall here briefly the basic concepts of elasticity in 3 dimensions. The reader can refer to [3] for further details on the elasticity theory. Let $\Omega_{0}$ be the initial configuration of the elastic template, the deformation is described by the Green-Lagrange strain tensor which is linearized under the small deformation assumption. We denote by $\epsilon(v)=\frac{1}{2}\left(\nabla v+\nabla v^{T}\right)$ the strain tensor and by $\sigma(v)=\lambda \operatorname{Tr}(\epsilon(v)) \Pi \mathrm{I}+2 \mu \epsilon(v)$ the stress tensor as functions of the displacement $v$. The coefficients $\lambda$ and $\mu$ stand for the Lamé coefficients (see [3]). It is well-known that the equilibrium position of an elastic body corresponds to 
the minimum of the elastic energy:

$$
F(v)=\frac{1}{2} \int_{\Omega_{0}} \sigma(v): \epsilon(v) d x
$$

Let $\mathbf{t}$ be the external superficial forces which deform the template. The force field $\mathbf{t}$ is derived from the M.R. volume data set according to the Gradient Vector Flow method [5]. The external energy is defined as work produced by the force field $\mathbf{t}$ due to the deformation. The following hypotheses for the field $\mathbf{t}$ are convenient for analyzing the image segmentation process.

- H1 The function $\mathbf{t}$ is defined on $\mathcal{O} ; \overline{\Omega_{0}} \subset \mathcal{O}, \mathbf{t} \in$ $C^{1}\left(\mathcal{O} ; \mathbb{R}^{3}\right)$ and its derivative $D \mathbf{t}$ is bounded on $\mathcal{O}$.

As mentioned in the introduction, the zero level of the external energy is reached when the boundary of the template corresponds to the borderline of the structure to be detected in the image. This implies that $D \mathbf{t}$ is not a regular linear operator on the boundary of the deformed template.

We denote by $H^{1}\left(\Omega_{0}\right)$ the classical Sobolev space of functions in $L^{2}\left(\Omega_{0}\right)$ with a derivative in distributional sense in $L^{2}\left(\Omega_{0}\right)$.

$$
H^{1}\left(\Omega_{0}\right)=\left\{\varphi \in L^{2}\left(\Omega_{0}\right) ; D \varphi \in L^{2}\left(\Omega_{0}\right)\right\}
$$

(see [3]) and we set $\mathbf{H}=\left(H^{1}\left(\Omega_{0}\right)\right)^{3}$.

Let $R=\operatorname{Ker} \epsilon$ be the subspace of rigid motions, define $\mathbb{H}=\left(H^{1}\left(\Omega_{0}\right) / R\right)^{3}$ equipped with the semi norm $\|\epsilon(v)\|_{L^{2}\left(\Omega_{0}\right)}$ which, thanks to Korn inequality, is a Hilbert space, and we denote by $\mathbb{H}^{\frac{1}{2}}$ the set of traces on $\partial \Omega_{0}$ of functions of $\mathbb{H}$. Finally, let $C$ denote the subset of admissible displacements: $C=\{v \in \mathbb{H}, \mathbf{t}(I+v)=0\}$ which is closed. Let $\mathbb{F}: \mathbb{H} \rightarrow \mathbb{R}$ be the strictly convex $\mathbb{H}$-coercive function defined by

$$
\mathbb{F}(v)=\frac{1}{2} \int_{\Omega_{0}} \sigma(v): \epsilon(v) d x .
$$

The elastic model for image segmentation reads: find $u$ verifying

$$
\mathbb{F}(u)=\inf _{v \in C} \mathbb{F}(v),
$$

the image of the heart $\tilde{\Omega}$ is recovered with $\tilde{\Omega}=(I+u) \Omega_{0}$.

Theorem 1: Problem 3 has a solution $u \in C$. Moreover if we assume that the derivative $D \mathbf{t}(\mathbf{I}+\mathbf{u})$ is a surjective operator belonging to $\mathcal{L}\left(\mathbb{H} ; \mathbb{H}^{1 / 2}\right)$, then we have the following optimality conditions: $\exists(\lambda, u) \in\left(\mathbb{H}^{1 / 2}\right)^{\prime} \times \mathbb{H}$ be such that

$$
\left\{\begin{array}{l}
-\operatorname{div}(\sigma(u))=0 \quad \text { in } \Omega_{0} ; \\
\sigma(u) \cdot n=-\lambda \circ D \mathbf{t}(I+u) \quad \text { on } \partial \Omega_{0} ; \\
\left.\mathbf{t}(I+u)\right|_{\partial \Omega_{0}}=0,
\end{array}\right.
$$

Remark 1: Thanks to the third equation in (4), the second equation in (4) can be expressed as:

$$
\sigma(u) \cdot n=\mathbf{t}(I+u)-\lambda \circ D \mathbf{t}(I+u) \quad \text { on } \partial \Omega_{0}
$$

\subsection{An algorithm for computing the mini- mizer}

The issue of this section is to propose an implementable algorithm. The convergence of this algorithm will be addressed in the next section. When the subset $C$ is not convex the difficulties for solving Problem 4 are twofold: solving $\mathbf{t}(I+u)=0$ and computing the Lagrange multiplier $\lambda$. As has been mentioned in remark 1 the second equation of the optimality conditions can be modified. Therefore we first propose to replace the equation $\left(4^{2}\right)$ by

$$
\sigma(u) \cdot n=\mathbf{t}(I+u)-\lambda \circ D \mathbf{t}(I+u) \quad \text { on } \partial \Omega_{0} .
$$

Problem 4 with equation $\left(4^{2}\right)$ replaced by the equation (6) is still a nonlinear problem. So we introduce the following iterative algorithm. Starting from $u_{0}=0$ and for a given $u_{k}, \operatorname{div}\left(\sigma\left(u_{\mathrm{k}}\right)\right)=0$ find $\left(\lambda_{k+1}, u_{k+1}\right) \in\left(\mathbb{H}^{1 / 2}\right)^{\prime} \times \mathbb{H}$ verifying

$$
\left\{\begin{array}{l}
-\operatorname{div}\left(\sigma\left(\mathrm{u}_{\mathrm{k}+1}\right)-\sigma\left(\mathrm{u}_{\mathrm{k}}\right)\right)=0 \quad \text { in } \Omega_{0} \\
\sigma\left(u_{k+1}\right) \cdot n=\mathbf{t}\left(I+u_{k}\right)-\lambda_{k+1} \circ D \mathbf{t}\left(I+u_{k}\right) \\
\mathbf{t}\left(I+u_{k+1}\right)=0 \quad \text { on } \partial \Omega_{0} .
\end{array}\right.
$$

To avoid computing the Lagrange multiplier $\lambda_{k+1}$, we note that if Algorithm 7 converges, then

$$
\sigma\left(u_{\infty}\right) \cdot n=-\lambda_{\infty} \circ D \mathbf{t}\left(I+u_{\infty}\right),
$$

thus we replace $-\lambda_{k+1} \circ D \mathbf{t}\left(I+u_{k}\right)$ by $\sigma\left(u_{k}\right) \cdot n$ and equation $\left(7^{3}\right)$ is cancelled because this condition is recovered implicitly for the limit $u$. In order to avoid discussing if $\mathbf{t}(I+u)$ is equilibrated or not, for $0<\beta$ a fixed parameter, we add the term $\beta\left(u_{k+1}-u_{k}\right)$. The algorithm 7 becomes: starting from $u_{0}=0$ and for a given $u_{k}, \operatorname{div}\left(\sigma\left(u_{\mathrm{k}}\right)\right)=0$ find $u_{k+1} \in \mathbf{H}$ verifying:

$$
\left\{\begin{array}{l}
-\operatorname{div}\left(\sigma\left(u_{\mathrm{k}+1}-\mathrm{u}_{\mathrm{k}}\right)\right)=0 \quad \text { in } \Omega_{0} \\
\sigma\left(u_{k+1}\right) \cdot n+\beta\left(u_{k+1}-u_{k}\right)=\mathbf{t}\left(I+u_{k}\right)+ \\
\sigma\left(u_{k}\right) \cdot n \quad \text { on } \partial \Omega_{0},
\end{array}\right.
$$

We obtain.

Lemma 1: For $u_{k} \in \mathbf{H}$ given, the problem 9 has a unique solution $u_{k+1} \in \mathbf{H}$.

Let us check that if the algorithm 9 converges then the limit function $\tilde{u}$ verifies the constraint: $\mathbf{t}(I+\tilde{u})=0$.

Lemma 2: Assume there exists a sequence $\left\{u_{k}\right\}_{k=1}^{\infty} \subset$ $\left.\left(H^{1}\left(\Omega_{0}\right)\right)^{3}\right)$ of solutions to the algorithm 9 which weakly converges towards $\tilde{u}$. Then $\tilde{u}$ verifies:

$$
\left\{\begin{array}{l}
-\operatorname{div}(\sigma(\tilde{\mathbf{u}}))=0 \quad \text { in } \Omega_{0} ; \\
\sigma(\tilde{u}) \cdot n=-\beta(\tilde{u})+\sum_{l=0}^{\infty} \mathbf{t}\left(I+u_{l}\right) \quad \text { on } \partial \Omega_{0} ; \\
\left.\mathbf{t}(I+\tilde{u})\right|_{\partial \Omega_{0}}=0,
\end{array}\right.
$$

Now let us specified the relation between Problem 10 and Problem 4. If we assume $D \mathbf{t}^{*}(I+\tilde{u})$ to be surjective, by 
defining $\mu \in D \mathbf{t}^{*}(I+\tilde{u})^{-1}\left[\beta(\tilde{u})-\sum_{l=0}^{\infty} \mathbf{t}\left(I+u_{l}\right)\right]$ the equation $\left(10^{2}\right)$ can be rewritten as

$$
\sigma(\tilde{u}) \cdot n=-\mu \circ D \mathbf{t}(I+\tilde{u}) .
$$

In [4] a similar algorithm has been implemented for a sphere-cube test case. The equation $\left(9^{2}\right)$ was replaced by $\sigma\left(u_{k+1}\right) \cdot n=\mathbf{t}\left(I+u_{k}\right)$ and the problem approximated with a tetrahedral finite element method of order one. This algorithm has been tested in [2]. Unfortunately, the constraint $\mathbf{t}(I+u)=0$ was not verified (see Figure 1 right).

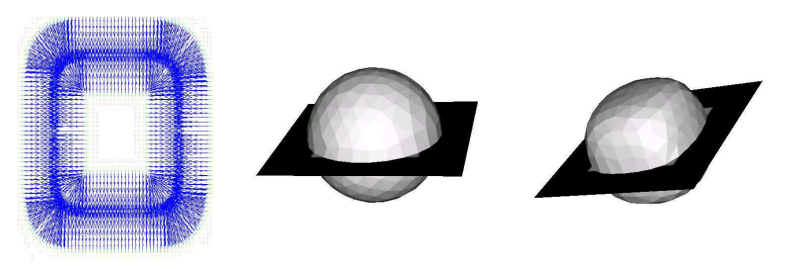

Figure 1. Left: a plane section of force field $\mathbf{t}$, center: initial template right: segmented data without constraint.

\subsection{Convergence of the algorithm in a fi- nite dimensional subspace}

In what follows, first we are given sufficient conditions for proving the convergence of the algorithm 9 when the space $\mathbf{H}$ is approximated with a finite dimensional subspace still denoted by $\mathbf{H}$, according to a Galerkin procedure. Then we are going to deal with a toy problem that mimics the main difficulties of the original problem (i.e. $D \mathbf{t}$ is not regular), for which we will exemplify the convergence of the algorithm 9 . Hereafter, we still denote by $u$ a solution to problem 3 in the approximated space $C \cap \mathbb{H}$. - Hypothesis $\mathbf{H 2}{ }^{1}$ Let $u$ be a solution to problem 3 in $C \cap$ $\mathbb{H}$, then the derivative $D \mathbf{t}(I+u)$ is semi negative definite, for all $\varphi \in \mathbf{H}$,

$$
(D \mathbf{t}(I+u) \varphi, \varphi)_{\partial \Omega_{0}} \leq 0 .
$$

So for arbitrary small positive parameter $\tau, \mathbf{t}^{\tau}=\mathbf{t}-\tau \mathbf{I}$ has a negative definite derivative.

In what is following, we will call problem 9, the problem which consists in finding $u_{k+1}$ when $u_{k}$ is given in the algorithm 9.

Now we are in position to give the main theorem for convergence of the algorithm 9.

Theorem 2: Let $\beta$ verifying $\left\|D\left(\mathbf{t}^{\tau}(I+u)\right)\right\|_{\mathcal{L}}<\beta$ be given, and assume the hypotheses $\mathrm{H} 1$ and $\mathrm{H} 2$ are satisfied, and that $D \mathbf{t}^{\tau}$ is locally a Lipchitzian function. Then there exists a neighborhood $\mathcal{U}$ of $u$ in $\mathbf{H}$ such as for all $u_{0} \in \mathcal{U}$, $\operatorname{div}\left(\sigma\left(\mathrm{u}_{0}\right)\right)=0$ the sequence of functions $\left\{u_{n}\right\}_{n \in \mathbb{N}} \subset \mathbf{H}$ verifying

$$
\begin{aligned}
& a\left(u_{n+1}, \varphi\right)+\beta\left(u_{n+1}, \varphi\right)_{\partial \Omega_{0}}= \\
& a\left(u_{n}, \varphi\right)+\beta\left(u_{n}, \varphi\right)_{\partial \Omega_{0}}+\left(\mathbf{t}^{\tau}\left(I+u_{n}\right), \varphi\right)_{\partial \Omega_{0}} \quad \forall \varphi \in \mathbf{H} .
\end{aligned}
$$

converges towards $\tilde{u}$ a solution to problem 3 in $C \cap \mathbf{H}$.

\section{Results}

Let $x \in \mathbb{R}^{3}$ the euclidean norm which is denoted by $\|x\|$, and $R_{f}$ a positive number. We choose as external force field the following function:

$$
\mathbf{t}(x)=-\alpha\left(\|x\|-R_{f}\right) \frac{x}{\|x\|} .
$$

with $0<\alpha<1$.

Now we would like to investigate the convergence property of the algorithm 9 for such an external field force. Set $R_{f}=\frac{1}{2}, R_{f}<R_{0}$ and let $u \in\left(\left(H^{1}\left(B_{R_{0}}\right)\right)^{3}\right)$ be solution to problem 3 , concerning the derivative of the operator $\mathrm{t}$ we have:

Lemma 3: The derivative $D \mathbf{t}(I+u)$ of the operator $\mathbf{t}(I+u)$ is defined by: for all $v \in\left(H^{1}\left(B_{R_{0}}\right)\right)^{3}$

$$
\begin{aligned}
& <D \mathbf{t}(I+u) \varphi, v>_{H^{\frac{1}{2}}, H^{-1 / 2}}= \\
& -\alpha \int_{\partial B_{R_{0}}} \frac{((I+u)(x) / \varphi(x))_{\mathbb{R}^{3}}((I+u)(x) / v(x))_{\mathbb{R}^{3}}}{\|(I+u)(x)\|_{\mathbb{R}^{3}}^{3}}+ \\
& \left(1-\frac{1}{2\|(I+u)(x)\|_{\mathbb{R}^{3}}}\right)(\varphi(x) / v(x))_{\mathbb{R}^{3}} d s .
\end{aligned}
$$

Since $u$ is a solution to problem 4 with $\Omega_{0}=B_{R_{0}}$, we have $\|(I+u)\|_{\mathbb{R}^{3}}=\frac{1}{2}$ a.e. on $\partial B_{R_{0}}$. Then the equation (14) writes: for all $v \in\left(H^{1}\left(B_{R_{0}}\right)\right)^{3}$

$$
\begin{aligned}
& <D K(u) \varphi, v>_{H^{\frac{1}{2}}, H^{-1 / 2}}= \\
& -\alpha \int_{\partial B_{R_{0}}} \frac{((I+u)(x) / \varphi(x))_{\mathbb{R}^{3}}((I+u)(x) / v(x))_{\mathbb{R}^{3}}}{\left(\frac{1}{2}\right)^{3}},
\end{aligned}
$$

which proves that the bilinear form induced by $D K(u)$ is semi negative definite.

\subsection{A toy 2D problem}

In order to evaluate practically, the speed of convergence of algorithms of type algorithm 9, we consider a set of points in $\mathbb{R}^{2}$ which are linked to each other with springs the elasticity constants are constants. The coordinates of the points are represented by a vector $u_{n}$, and an extreme simplification of the elastic model is represented by a diagonal with an upper and a lower diagonal matrix definite positive $A^{-1}$. The function $\mathbf{t}$ is define for all $x \in \mathbb{R}^{2}$ with $\alpha=.2$ by:

$$
\mathbf{t}(x)=-\alpha(\|x\|-1) \frac{x}{\|x\|} .
$$


Please note that if $L^{-1}$ sands for the operator which to $u_{k}$ associates $u_{k+1}$ solution to problem 9 , then algorithm 9 can be written as:

$$
u_{n+1}=u_{n}+L^{-1}\left(\begin{array}{l}
0 \\
\mathbf{t}\left(I+u_{n}\right)
\end{array}\right) .
$$

So the simplified version for the toy $2 \mathrm{D}$ problem reads:

$$
u_{n+1}=u_{n}+A^{-1} \mathbf{t}\left(I+u_{n}\right)
$$

The three following figures depict the evolution of the springs, the balls with red lines represent the initial configuration. The first picture is the configuration after 2 iterations where green squares are the points and blue lines the springs. The second picture is the configuration after 30 iterations and the third one represents the configuration after 3 iterations for a double value of the elasticity constant.
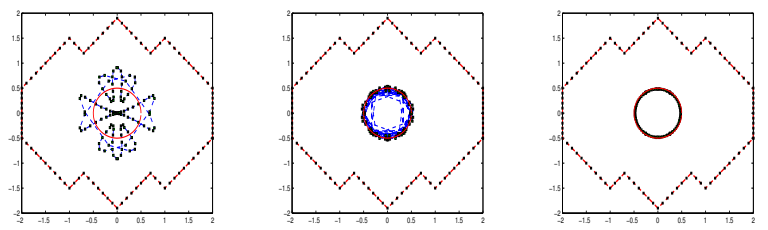

Figure 2. Left: 2 iter, center: 30 iter right: 3 iter with a double value of elasticity constant.

\section{Discussion and conclusions}

We end this article, with the sphere-cube test case. The space $\mathbf{H}$ is approximated by a tetrahedral finite element method of order one. For the numerical example presented here, we have used 2237 tetrahedra and 519 nodes. In Figure 1 on the left we have a section of the force field $\mathbf{t}$, the initial template $\Omega_{0}$ is a sphere and is depicted on the center. The convergence for the algorithm 9 is reached after 10 iterations and $\tilde{\Omega}$ the segmented image is presented in Figure 4 on the left. Finally, In Figure 4 on the right we display medical data of the heart of a patient segmented with algorithm 9 at the end of diastole (from [4] for the data).
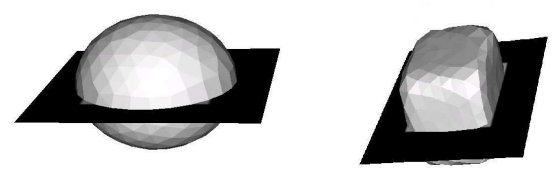

Figure 3. Left: initial sphere, right deformed configuration from a sphere with Algorithm 9.
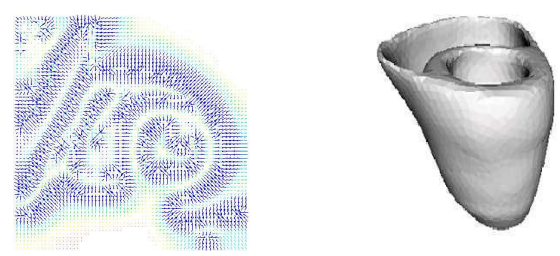

Figure 4. Left: a section of the force field $\mathbf{t}$, right medical data segmented with Algorithm 9.

We have proved that the segmentation problem for cardiac M.R.I is mathematically well posed, and we have proposed a simple algorithm proved to be convergent for computing the solutions. The formulation of the problem as a constraint problem have been demonstrated a necessary condition for the segmentation in cardiac M.R.I. The algorithm proposed is simple and converges with few iterations and ensure to reach the zero level of the external energy with an arbitrary large precision corresponding to an arbitrary small parameter $\tau$ in $\mathbf{H} \mathbf{2}$.

The next step for improving the segmentation algorithm 9 is to eliminate the hypothesis of small displacements. This means in particular that we do not require $\Omega_{0}$ to be in a neighborhood of $\tilde{\Omega}$, while the image still is recoverable. To do this we propose using an incremental method combined with dynamic meshing (see [2]), or nonlinear elastic models.

\section{References}

[1] Sermesant M, Forest C, Pennec X, Delignette H, Ayache N. Deformable biomechanical models: Application to 4D cardiac image analysis. Medical Image Analysis 2003; 7.

[2] Baker TJ, Pebay P, Pousin J. Dynamic meshing for finite element based segmentation of cardiac imagery. WCCM V - Fifth World Congress on Computational Mechanics, Vienna, 2002.

[3] Ciarlet PG. Mathematical elasticity II, theory of plates. Studies in Mathematics and its Applications 27. 1997; 27. Amsterdam:Elsevier, 1997.

[4] Pham QC. Segmentation et mise en correspondance en imagerie cardiaque multimodale conduites par un modèle anatomique bi-cavités du coeur. PhD Dissertation, INPG, 2002.

[5] Xu C, Prince JL. Snakes, shapes, and gradient vector flow. IEEE Trans Image Processing 1998; 7(3): 359-369.

Address for correspondence:

jerome Pousin

Institute C. Jordan INSA de Lyon, 20 avenue A. Einstein, F69621 Villeurbanne, France

jerome.pousin@insa-lyon.fr 